\title{
LA PEDAGOGÍA EN EL CURRICULUM ACADÉMICO
}

\section{Comisión de investigaciones pedagógicas de la UPS}

\section{Presupuestos críticos y epistemológicos}

Obligado es comenzar precisando que a diferencia del concepto general de educación, cuya significación puede ir más allá de la educación del niño y del joven, y que tampoco es exclusiva de la escuela o el colegio (ya que también los padres educan junto con otras instituciones, organismos y procesos sociales que tienen competencias y efectos educativos), definimos la pedagogía como la específica formación o educación del niño, que también específicamente tiene que ver con su desarrollo intelectual ${ }^{1}$. En este sentido la pedagogía supone no sólo una ciencia del niño, sino también de manera más precisa una ciencia del desarrollo del pensamiento y conocimiento en el niño ${ }^{2}$. Pero, puesto que la pedagogía no es sólo una ciencia, sino también una práctica podría ser definida en su específica epistemología como una teoría del aprendizaje del niño y una práctica de su enseñanza, considerando que es la teoría del aprendizaje que funda, re-

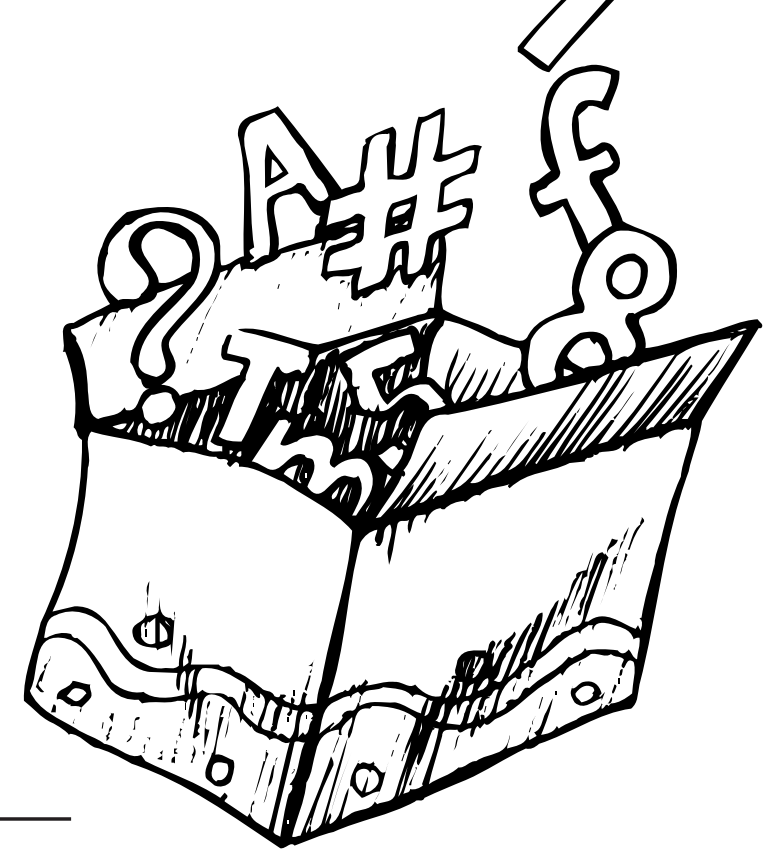

* Este texto tiene como base una investigación sobre los currículum de la carrera de pedagogía de la Universidad Politécnica Salesiana, realizada en el año 2004 por la comisión integrada por María Elena Ortiz, Sebastián Granda, Jaime Padilla y José Sánchez Parga, quien coordinó el estudio y redactó el presente texto.

1 Según la etimología el griego pedagogein significa "instruir al niño", verbo del que Platón presenta el interesante significado de "seguir, paso a paso, como se sigue a un niño" (Alcibíades, 135 a). Cfr. A. Bailly, Dictionnaire Grec - Francais, Hachette, Paris, 1950.

2 Paideia significa en griego tanto la "instrucción de los niños", como la "cultura" no tanto como contenido sino como acción, pero también el conocimiento como contenido y acción. 
gula y dirige, en definitiva racionaliza, la práctica del aprendizaje.

Se puede extender la pedagogía también al aprendizaje y enseñanza del adolescente (a partir de los 14 años), en la medida que la evolución del pensamiento del niño, el desarrollo de su inteligencia y fases de su aprendizaje son prolongación y transformación de los del niño. Hay, sin embargo, que precisar una diferencia cualitativa muy importante: mientras que el aprendizaje del niño (su evolución, fases y formas de su desarrollo) condicionan y pautan el proceso y procedimiento de enseñan$z a$, adaptándose ésta como un apoyo a aquel, en el caso del adolescente, es la organización y secuencia propias de los conocimientos enseñados (historia, geografía, matemáticas...), los que contribuyen a formar e in-formar el aprendizaje joven.

Según esto la pedagogía en cuanto Ciencia tiene por objeto el proceso de enseñanza-aprendizaje del conocimiento por parte del niño. Pero precisando que la pedagogía se interesa directa, prioritaria y específicamente en el aprendizaje del niño y sólo consecuentemente y como parte de ello en la enseñanza asociada a dicho proceso de aprendizaje. En otras palabras, y a diferencia de la corriente más convencional o tradicional, que siempre ha priorizado la ense- ñanza (del maestro) sobre el aprendizaje del niño, haciendo de este la consecuencia de aquélla, hay que partir de la "nueva pedagogía" (aunque no sea tan nueva y más bien responda a su concepción más clásica), la cual se funda: a) sobre el primado del aprendizaje del niño, al que se adapta la enseñanza del maestro; b) sobre una concepción del conocimiento no tanto en sus contenidos (los que se transmiten por parte del maestro), sino, sobre todo en cuanto acción de conocer (por parte del niño), la cual se desarrolla de acuerdo a una secuencia de fases y formas cada vez más elaboradas, condicionadas por un determinado modelo pedagógico.

Una constatación preliminar, sujeta a ulteriores y más documentadas verificaciones, pone de manifiesto un generalizado modelo educativo vigente, que junto a un relativo desconocimiento del evolucionismo, de la psicología genética y de las ciencias del conocimiento (la clásica "psicología racional”, gnosología o neurociencias) han impedido entre otros factores que se desarrollara un pensamiento pedagógico.

Hay una estrecha correspondencia entre el modelo pedagógico (o pedagogías del conocimiento), el modelo educativo y un determinado modelo de compresión y tratamiento del niño y de las condiciones de la in- 
fancia en una sociedad. Un modelo pedagógico que privilegia de manera predominante la enseñanza sobre el aprendizaje, la acción del maestro sobre la del alumno o estudiante, la transmisión de informaciones y conocimientos, se encontrará estrechamente asociado a un modelo educati$v o$, que, a su vez, enfatiza la acción y las intervenciones de la sociedad, de las instituciones y procedimientos instruyéndolo, informatizándolo, disciplinándolo, que en definitiva coarta más que coaccionar su propio devenir adulto. Ambos modelos, pedagógico y educativo, condicionarán a su vez todo el conjunto de representaciones y relaciones de una sociedad y sus niños.

Por el contrario, un modelo pedagógico, basado sobre el desarrollo de la inteligencia del niño (el que dentro de las pedagogías del conocimiento sería un modelo "autoestructuracionista" o "interestructuracionista", según Louis Not), sobre el conocimiento del niño en cuanto acción, daría lugar a un modelo educativo que respetaría mucho más la autonomía y creatividad del niño; la sociedad lejos de imponer los modelos adultos en sus representaciones y relaciones con los niños, más bien permitirían que estos en su devenir adulto produjeran su propio modelo adulto.
Históricamente los modelos pedagógicos y educativos se encontraban muy condicionados por el modelo de sociedad. El problema hoy se plantea de otra manera por dos razones fundamentales: si bien, por un lado, se conoce perfectamente cómo se desarrolla y se forma la inteligencia del niño, cuáles son las fases y formas en la evolución de su pensamiento, así como las condiciones, dispositivos y procesamientos que contribuyen a su organización y orientación intelectual, de otro lado, la sociedad moderna dispone de unas fuerzas y recursos en su producción de sociedad mucho más efectivos, y por consiguiente capaces de determinar el proceso de educación del niño.

Ya las sociedad más antiguas (Mesopotamia y Egipto) se habían interesado por la educación, pero sólo la Grecia clásica hace de la educación una reflexión sostenida y sistemática cada vez más compleja y especializada, donde comienzan a diferenciarse dos posiciones, las cuales atravesarán después la larga historia del pensamiento pedagógico y educativo. La época heroica se mostró reacia a toda educación, bajo el presupuesto de que con ella se impediría la creatividad de las nuevas generaciones y el rejuvenecimiento de la sociedad, ya que todo proceso edu- 
cativo se volvería cada vez más formulario y formalista; para aquella época pre-pedagógica o anti-pedagógica, que apostaba, más bien, a una suerte de "socialización" del niño suficientemente educativa por sí misma, las decadencias conllevan siempre un progreso educacional o un exceso de educación ${ }^{3}$.

Con Sócrates se habría inaugurado la culminación del afán educativo en Atenas, al consagrar con la educación un doble proceso: racionalizar el desarrollo de la enseñanza y aprendizaje de conocimientos para contribuir a la formación del ciudadano. Pero también entonces se inicia la evolución degenerativa de una ciencia que terminaría reducida a una "pedagogía como doctrina" (Tovar, p. 220) y a una pedagogía utilitaria y mercantil como fue la de los sofistas; en definitiva una pedagogía deformadora, más racionalista que racional y "superpuesta" a los reales procesos de aprendizaje del niño y del adulto ${ }^{4}$.
Pero incluso la educación socrática presentaba un límite y corrección a los "excesos y pedanterías" de los pedagogos profesionales. Nada casual, por ello que dentro de las grandes corrientes de las "pedagogías del conocimiento", Sócrates sea colocado dentro de las "pedagogías heteroestructuracionistas" (Louis Not), pero con una variante "coactiva", que racionalizando la tarea mayéutica del maestro, presupone la actividad e iniciativa intelectuales del conocimiento del alumno 5 .

\section{El desarrollo de la pedagogía como enseñanza y aprendizaje}

Todo el desarrollo de la pedagogía a lo largo de su historia ha sido en gran medida y de manera predominante el desarrollo de la enseñanza y aprendizaje de los conocimientos. Lo que Not denomina la "heteroestructuración del conocimiento", y que

3 Antonio Tovar se refiere a la profunda idea de educación, que tenía el rey bárbaro de permitir la educación de sus hijas, pero no de sus hijos, pues quien temblaba ante un maestro no podía ser un guerrero con su valor intacto (Vida de Sócrates, Ed. Alianza, Madrid, 1986: 205).

4 "Una deformación del hombre, metido en un currículo de reducidísimo horizonte, una orientación retórica y practicista, que llevaba la intervención racional a los senos más profundos, fértiles y misteriosos de la persona humana" (Tovar, p. 220).

5 "Si Sócrates hubiera tenido la experiencia que da nacer después, no habría creído ni un momento en la educación. Esa contradicción que hallamos entre su fe en la educación y su respeto a los espontáneo y heredado, se explica y resuelve sin más que pensar que para Sócrates la educación no era todavía esa tremenda intervención en lo más secreto del alma humana en que se convirtió muy pronto" (Tovar, p. 222). 
presupone una concepción pseudoidealista del conocimiento, según la cual las ideas, los conceptos y los conocimientos pueden ser enseñados y transmitidos y por consiguiente aprendidos. Como si las ideas, los conceptos y los conocimientos fueran simples informaciones y datos que son objeto de comunicación entre un emisor y un receptor. Cuando en realidad las ideas, los conceptos y los conocimientos no pueden ser en realidad enseñados ni aprendidos; lo que se enseña y se aprende son datos, informaciones y mensajes, pero no ideas, conceptos y conocimientos; estos sólo pueden ser objeto de explicación y de comprensión; es decir, resultado de una doble actividad del pensamiento. Según esto las informaciones y datos, los hechos y mensajes que son comprendidos y explicados se convierten en conocimientos, ideas y conceptos.

Un hecho o un conocimiento sólo es explicado en la medida que es comprendido, ya que sin una real comprensión no hay una efectiva explicación. De igual manera que un hecho o conocimiento sólo es comprendido realmente en la medida que puede ser explicado. En conclusión, no hay conocimientos en cuanto contenidos sin un conocimiento en cuanto acción de conocer: es esta actividad del conocimiento que no es simplemente la memoria del aprender la que hace posible el comprender.

Por consiguiente, en contra de quienes sostienen que primero se aprende y después se comprende, es necesario comprender para poder después aprender lo comprendido.

Las pedagogías del conocimiento "autoestructuracionistas" se fundan sobre una teoría del conocimiento o gnoseología de presupuestos empiristas, según la cual el desarrollo de la inteligencia se opera a partir de la experiencia sensible 6 . Una pedagogía del conocimiento basada en tales presupuestos dejaría que todo el desarrollo intelectual y del conocimiento del niño se operara a partir de todo un conjunto diversificado de experiencias dispuesto y organizado por el maestro o la institución pedagógica. Cuando nunca la experiencia por sí sola es capaz de transformarse en conocimiento, dando cuenta de sus propias causas, razones y lógicas que la han producido. Quien ha cobrado un salario, tiene la experiencia de lo que es el salario,

6 De acuerdo al principio tomista según el cual "nada hay en el intelecto que antes no pase por los sentidos" (nihil in intellectu quod prius non fuerit in sensu). 
pero no conoce lo que es el salario, ignora las causas, las razones y lógicas que lo producen y permiten comprender su sentido.

Las necesidades y urgencias educativas de la sociedad, un modelo de relación con el niño y de tratamiento de la educación infantil, y, finalmente, el desconocimiento de las principales corrientes del pensamiento pedagógico que se desarrollan a mediados del siglo XX en torno a la escuela piagetiana, todo ello parece haber contribuido a impedir que se formará un pensar educativo en el país. Ya Piaget advertía este fenómeno desde su propio campo psico-pedagógico, y un filósofo como Ortega y Gasset lo destacaba en su doble dimensión histórica y actual: "descuido en el que andan las ideas pedagógicas de nuestro tiempo"; "anacronismo constitucional que suele padecer el pensamiento pedagógico"; "el pedagogo no ha sido nunca el filósofo de su pedagogía" 7 .

Para Piaget el defecto de "una ciencia de la educación suficientemente elaborado" se debe a un excesivo "empirismo de la pedagogía", y su instrumentalización didáctica; y se pregunta por qué la masa de educadores en el mundo "no genera una elite de investigadores que hagan de la pedagogía una disciplina a la vez científica y viva como todas las disciplinas aplicadas, que participan a la vez del arte y de la ciencia”. Y, aunque, no deja de reconocer que la pedagogía "es una ciencia entre otras incluso muy difícil por la complejidad de factores en juego", tampoco omite señalar en qué medida las ingenierías educativas y las preocupaciones metodológicas han constituido el más serio obstáculo para el pensamiento pedagógico y su desarrollo teórico y científico ${ }^{8}$.

Pero el mayor fracaso pedagógico ha consistido en compensar con creces la ignorancia de cómo aprende el niño y cómo se desarrolla su inteligencia con una excesiva preocupación por cómo enseñarle conocimientos, cuando nada se puede real y efectivamente enseñar a un niño sin saber cómo el niño aprende. Una pedagogía sólo es coherente y eficiente "si se pone el énfasis sobre las facultades de iniciativa y de invención (del niño) más que sobre la acumulación de saberes "a disposición de todos los manuales" (Piaget, o.c., p. 132).

\footnotetext{
7 J. Ortega y Gasset, "Pedagogía y anacronismo (1923)", en Obras Completas, t. III, Revista de Occidente, 1947:57.

8 Cfr. Jean Piaget, Psychologie et Pédagogie, Densel / Gontier, Sarthe, $1969: 20-23$.
} 


\section{Hacia una definición teórico-científica de la pedagogía}

Toda teoría pedagógica presupone siempre de manera más o menos explícita una teoría del aprendizaje, la cual, a su vez, se funda en una teoría del conocimiento también más o menos implícita. Las pedagogías que basan el aprendizaje en la enseñanza y que hacen de ésta el principio y motor de todo aprendizaje presuponen una concepción empirista y funcionalista o conductista del aprendizaje (a lo Skinner), la que Popper denominó "la teoría del cubo vacío de la inteligencia" (the empty bucket theory of mind).

La pedagogía en su núcleo científico comporta una teoría (comprensión-explicación) de cómo se forma y organiza la inteligencia humana, como se desarrolla en sus sucesivas fases o estructuras secuenciales desde los primeros años del niño, cómo evoluciona el aprendizaje en el niño, y finalmente, cómo se construye una teoría pedagógica de la enseñanza en apoyo y estrecha correspondencia ("interestructuracionista") con el proceso de aprendizaje. Según esto la pedagogía se constituye en cuanto espacio científico interdisciplinar, en el que convergen una gnoseología o epistemología y una psicología del desarrollo del niño.

La formación y desarrollo del conocimiento en el niño posee un carácter secuencial, lo cual implica una evolución por fases o estadios, todos necesarios y cada uno de resultante necesariamente del anterior (salvo el primero) y preparación del siguiente (salvo el último)9. Sólo la comprensión de este proceso, que marca las condiciones y fases del desarrollo del pensamiento en el niño y de su aprendizaje, permitirá construir una pedagogía que contribuya a tal desarrollo.

Es importante notar que: a) la sucesión de conductas mentales es siempre constante, independiente de las aceleraciones y retrasos, que puedan modificar las edades cronológicas; de ahí la necesidad de distinguir en psicología entre la edad temporal y la edad mental del niño (Piaget, o.c., p. 37, nota); b) cada estadio o fase se define no por una propiedad dominante, sino por una estructura del conjunto de las condiciones, disposiciones y conductas propias de dicho estadio; c) estas estructuras presentan un proceso de integración tal, que cada una está

9 Jean Piaget, Biologie et connaissence, Gallimard, Paris, 1967. 
preparada en la anterior y se integra en la siguiente ${ }^{10}$.

1. En la inteligencia humana hay un componente orgánico-hereditario: la inteligencia sensorial-motriz, cuyo componente hereditario comporta mucho más que el instinto, porque supone la transmisión de un funcionamiento susceptible de conducir muy lejos $\mathrm{y}$ de aprender indefinidamente, $\mathrm{y}$ mucho menos, porque no implica la transmisión de ninguna estructura fija particular.

Esta dimensión orgánica del conocimiento, que ni se adquiere ni se aprende, está integrada por esquemas cognitivos que derivan unos de otros, y que marcan la estrecha asociación entre la organización biológica y el conocimiento. Tal es el caso de la memoria y la lógica: se trata de los esquemas sensoriales y motores $\mathrm{u}$ operatorios, cuya conservación (función biológica) adopta en el hombre la forma la memoria y cuya organización (función biológica) se desarrolla en el hombre en forma de lógica.
Según esto los procesos cognitivos son resultado de una autorregulación orgánica y de las interacciones con el exterior, operando de manera análoga a los seres vivos: ambos presentan una organización que conserva asimilando las realidades exteriores, y cuya organización es autorreguladora. Por tal razón "las funciones cognitivas serían, en esta perspectiva, órganos especializados de la autorregulación de los intercambios en el seno del comportamiento" (p. 59). En otras palabras, "la primera función del conocimiento es ser una asimilación en el sentido preciso de una interacción entre el sujeto y el objeto, donde la mayor acomodación del sujeto a las características del objeto corresponde a un incorporación a las estructuras anteriores del mismo sujeto. En consecuencia el objeto del conocimiento nunca es independiente de las actividades cognitivas del sujeto; por ello la objetividad no es un estado sino un proceso, el que supone una concatenación de aproximaciones sucesivas, quizás nunca completa; tales aproximaciones que conducen al objeto no son simple-

10 No tiene mucho sentido pretender cuestionar la teoría general de Piaget desde determinados hallazgos experimentales (como el de Nina Talizina) ya que una teoría no es invalidada experimentalmente a no ser que las pruebas experimentales correspondan a un nuevo planteamiento teórico. De otro lado, no es muy pertinente referirse a cuestiones referirse a cuestiones disputadas de una ciencia en un currículo, que tiene una función operativa y de programación académica. 
mente de naturaleza aditiva (acumulación de informaciones), sino que comporta un proceso esencial de descentración o liberación de adherencias subjetivas; lo que se completa en la explicación del objeto; donde este adquiere la máxima objetividad.

Una pedagogía científica ha de descartar una concepción positivista de la inteligencia en cuanto facultad constitutiva de la naturaleza humana, y definitivamente dada en su mecanismo o funcionamiento formal (la lógica) para pensar la inteligencia en términos genéticos (filogenéticos, propios de la especie, y ontogenéticos propios del individuo): "la razón... reelabora por una sucesión de construcciones operatorias" (p. 118); sin éstas aquélla no es posible.

2. Las estructuras o esquemas cognitivos lógico-matemáticos ni son formas hereditarias del conocimiento (como el caso de la inteligencia sensorio-motriz) ni pertenecen a los conocimientos adquiridos, aunque son la condición necesaria para la formación de estos últimos. En la experiencia lógico-matemática los conocimientos obtenidos no son toma- dos directamente de los objetos como tales sino de las acciones u operaciones ejercidos sobre ellos (agrupándolos, por inclusión y clasificación, ordenándolos por seriaciones o relaciones...).

A diferencia del campo de la percepción, donde hay ciertas estructuras innatas, el conocimiento lógicomatemático se constituye a partir de una categoría a priori (en el sentido kantiano): por ejemplo la causalidad. La función simbólica (lenguaje, juego, imaginarios...) y las operaciones concretas (clasificación, seriación, correspondencias...) corresponden a esta fase del conocimiento y preparan el nivel superior de las operaciones proposicionales.

\section{El campo del conocimiento pedagógico}

Según N. Chomsky, y de acuerdo con Piaget, "es conveniente construir una teoría del aprendizaje particular para cada terreno del conocimiento" 11 . Por consiguiente, el área teórico-científica de la pedagogía debería abarcar el campo de conocimientos relativos a 
a) Biología y conocimiento.

Psicología de la inteligencia: el nacimiento de la inteligencia en el niño.

Psicología genética.

b) La representación del espacio en el niño.

El desarrollo de la noción del tiempo en el niño.

Génesis del número en el niño. El desarrollo de las cantidades en el niño.

c) Formación del símbolo en el niño. El juicio moral en el niño.

La representación del mundo en el niño.

d) De la lógica del niño a la lógica adolescente.

Transformaciones de las operaciones lógicas.

Educación e instrucción. Corrientes pedagógicas.

"Pedagogías del conocimiento" (Not).

Una vez que se conoce, se comprende y explica, la formación y desarrollo del conocimiento en el niño es posible elaborar una teoría de la enseñanza y de la didáctica en estrecha correspondencia con la evolución de la inteligencia del niño y su proceso de aprendizaje.

\section{De la pedagogía como ciencia al currículo académico}

El estudio de las investigaciones de fin de carrera o tesis de grado en la Facultad de Pedagogía demostró que sólo un número muy reducido de estos trabajos había tenido por objeto problemas o temas específicamente pedagógicos relativos al aprendizaje y enseñanza; la mayor parte de las investigaciones realizadas se referían a problemáticas más o menos periféricas o distantes de la pedagogía, relativas a la educación en general o a cuestiones educativas de la más diversa índole ${ }^{12}$. Como una forma de profundizar dicho estudio nos preguntamos por la causa de un tal resultado, y consideramos que sería necesario indagar las programaciones de la misma carrera de pedagogía.

Las conclusiones de este estudio condujeron a plantear la cuestión del currículo o programa de la carrera de pedagogía, y a preguntarse en qué medida la pedagogía como ciencia se encontraba expresa y específicamente definida como un campo particular del conocimiento, en el que se pudieran identificar un área o cuerpo teórico, "ciencia básica" de pedago-

12 Cfr. La publicación de un informe sobre esta investigación con el título "La investigación pedagógica en la Escuela de Pedagogía de la Universidad Politécnica Salesiana", Universitas, n. 5, diciembre 2004. 
gía, diferentes del área de especialización, de aplicación o extensión de la pedagogía con sus diferentes desarrollos y orientaciones.

Partimos del supuesto que todo currículum consiste en la transformación de la organización y desarrollo de una ciencia en una estructura y programación académicas orientadas a la formación científica y profesional. De ahí la necesidad de pensar ("pensum") la ciencia en su organización interna, en sus presupuestos teóricos, sus fases de desarrollo, sus campos de especialización y de aplicación de sus conocimientos, para darle la forma operativa de un programa académico de formación por materias. Es obvio que la función y finalidad del $\mathrm{cu}$ rrículo consistente en la formación científica y profesional del estudiante deberán incorporar no sólo las características de la sociedad sino también los posibles ámbitos profesionales y laborales, en los que los estudiantes deberán ejercer su formación científica y académica.

Según esto, es necesario analizar el currículo de pedagogía para comprobar en qué medida traduce y expresa la pedagogía en cuanto ciencia, o más exactamente la organización y desarrollo científicos de la pedagogía. En otras palabras, se trata de verificar qué concepción de la pedagogía, en cuanto ciencia, refleja y plas- ma el currículo o programa de la carrera de pedagogía. O formulado en otros términos ¿qué concepción de la ciencia pedagógica expresa o refleja el pensum de la carrera de pedagogía?

Un primer análisis de las "mallas" de la carrera de pedagogía de 1996 permitió diferenciar 5 áreas o campos de conocimiento, en los que se podían agrupar todas las materias de acuerdo al título de la materia y a la sucinta descripción de sus contenidos: pedagógicos, pedagogía considerada desde otras ciencias o campos de conocimiento, otras ciencias y campos de conocimiento diferentes de la pedagogía, materias de metodología y técnicas, materias experimentales y aplicadas.

Si se compara el programa de pedagogía del año 1996 con el del año 2002 , se observa un notable aumento del número de materias: de 42 a 56 (más del 30\%). Sin embargo, no se modifica el número de materias específicamente pedagógicas: 6 en ambos programas. En cambio casi se duplica el número de materias, que comprende el área de "otros campos de conocimiento" (pasa de 8 a 11), y el referido a enfoques de la pedagogía desde otras ciencias o campos de conocimiento (de 7 a 16 materias). Finalmente se reducen las materias (de 15 a 12) en el campo de las metodologías y técnicas, pero se duplican 


\section{Cuadro 1}

Mallas del año 1996

\begin{tabular}{|l|r|r|}
\hline EGB - Ciencias Sociales & \multicolumn{1}{c}{$N^{\circ}$} & $\%$ \\
\hline Pedagógicas & 4 & 9,3 \\
\hline P.Desde otro Campo & 4 & 9,3 \\
\hline Otros campos conoc. & 16 & 37,2 \\
\hline Metodologías y Técnicas & 14 & 32,6 \\
\hline Experimen. Aplicadas & 5 & 11,6 \\
\hline Total & $\mathbf{4 3}$ & $\mathbf{1 0 0}$ \\
\hline
\end{tabular}

\begin{tabular}{|c|c|c|}
\hline EGB - Cultura Estética & $\mathbf{N}^{\circ}$ & $\%$ \\
\hline Pedagógicas & 3 & 6,98 \\
\hline P. Desde otro Campo & 4 & 9,3 \\
\hline Otros campos conoc. & 16 & 37,2 \\
\hline Metodologías y Técnicas & 15 & 34,9 \\
\hline Experimen. Aplicadas & 5 & 11,6 \\
\hline Total & 43 & 100 \\
\hline
\end{tabular}

\section{EGB - Lenguaje y Com. № \%}

\begin{tabular}{|c|c|c|}
\hline Pedagógicas & 5 & 11,6 \\
\hline P. Desde otro Campo & 4 & 9,3 \\
\hline Otros campos conoc. & 15 & 34,9 \\
\hline Metodologías y Técnicas & 14 & 32,6 \\
\hline Experimen. Aplicadas & 5 & 11,6 \\
\hline Total & 43 & 100 \\
\hline
\end{tabular}

\begin{tabular}{|c|c|c|}
\hline EGB - Matemáticas & No & $\%$ \\
\hline Pedagógicas & 4 & 9,3 \\
\hline P. Desde otro Campo & 4 & 9,3 \\
\hline Otros campos conoc. & 16 & 37,2 \\
\hline Metodologías y Técnicas & 14 & 32,6 \\
\hline Experimen. Aplicadas & 5 & 11,6 \\
\hline Total & 43 & 100 \\
\hline
\end{tabular}

\begin{tabular}{|l|l|l|}
\hline Parvularia & No & $\%$ \\
\hline
\end{tabular}

\begin{tabular}{|l|r|r|}
\hline Pedagogía & \multicolumn{1}{c|}{$N^{\circ}$} & $\%$ \\
\cline { 2 - 3 } & \multicolumn{1}{|r||}{} & 14,3 \\
\hline Pedagógicas & 7 & 16,7 \\
\hline P.Desde otro Campo & 8 & 19 \\
\hline Otros campos conoc. & 15 & 35,7 \\
\hline Metodologías y Técnicas & 6 & 14,3 \\
\hline Experimen. Aplicadas & $\mathbf{4 2}$ & $\mathbf{1 0 0}$ \\
\hline \multicolumn{2}{|l|}{ Total }
\end{tabular}

\begin{tabular}{|l|r|r|}
\cline { 2 - 3 } & Pedagógicas & 6,98 \\
\hline P.Desde otro Campo & 4 & 9,3 \\
\hline Otros campos conoc. & 20 & 46,5 \\
\hline Metodologías y Técnicas & 13 & 30,2 \\
\hline Experimen. Aplicadas & 3 & 6,98 \\
\hline Total & $\mathbf{4 3}$ & $\mathbf{1 0 0}$ \\
\hline
\end{tabular}

Cuadro 2

Mallas del año 2002

\begin{tabular}{|l|r|r|}
\hline \multicolumn{1}{|l}{ Parvularia } & \multicolumn{1}{c}{ No } & $\%$ \\
\cline { 2 - 3 } & \multicolumn{1}{|r|}{} & 7,41 \\
\hline Pedagógicas & 10 & 18,5 \\
\hline P.Desde otro Campo & 14 & 25,9 \\
\hline Otros campos conoc. & 16 & 29,6 \\
\hline Metodologías y Técnicas & 10 & 18,5 \\
\hline Experimen. Aplicadas & 10 \\
\cline { 1 - 2 } Total & $\mathbf{5 4}$ & $\mathbf{1 0 0}$ \\
\hline
\end{tabular}

\begin{tabular}{|l|r|r|}
\hline Pedagogía & \multicolumn{1}{c}{$N^{\circ}$} & $\%$ \\
\hline \hline Pedagógicas & 6 & 10,7 \\
\hline P.Desde otro Campo & 16 & 28,6 \\
\hline Otros campos conoc. & 11 & 19,6 \\
\hline Metodologías y Técnicas & 12 & 21,4 \\
\hline Experimen. Aplicadas & 11 & 19,6 \\
\hline Total & $\mathbf{5 6}$ & $\mathbf{1 0 0}$ \\
\hline
\end{tabular}


aquellas de carácter más experimental y aplicado.

En conclusión, respecto del anterior programa de la carrera, el del año 2002 reduce las materias específicamente pedagógicas y las relativas a la pedagogía enfocadas desde otros campos de conocimiento, así como las metodológicas, mientras que aumenta materias de otros conocimientos sin relación con la pedagogía y las materias experimentales y aplicadas, pero cuya instrumentalización estaría más desprovista de conocimiento o pensamiento pedagógico.

\section{Cuadro 3}

Pedagogía 2002

\begin{tabular}{|c|c|c|c|c|c|c|}
\hline Nivel & Nombre de la asignatura & $\begin{array}{l}\text { Mat. } \\
\text { Pedag. }\end{array}$ & $\begin{array}{l}\text { Pedag. } \\
\text { Cien. }\end{array}$ & $\begin{array}{l}\text { Otras } \\
\text { Cienc. }\end{array}$ & $\begin{array}{l}\text { Metod. } \\
\text { Téc. }\end{array}$ & $\begin{array}{c}\text { Exp. } \\
\text { Aplicad. }\end{array}$ \\
\hline 1 & Sistema Educativo Salesiano & & $x$ & & & \\
\hline 1 & Visión retrospectiva de la educación & & $\mathrm{x}$ & & & \\
\hline 2 & Educación y Comunicación & & $\mathrm{x}$ & & & \\
\hline 2 & Familia y Educación & & $\mathrm{x}$ & & & \\
\hline 3 & Filosofía de la Educación & & $\mathrm{x}$ & & & \\
\hline 3 & Psicología del Aprendizaje & & $x$ & & & \\
\hline 3 & Sociología de la Educación & & $x$ & & & \\
\hline 4 & Epistemología de la Educación & & $x$ & & & \\
\hline 4 & Infopedagogía & & $\mathrm{x}$ & & & \\
\hline 5 & Nueva Sociología de la Educación & & $\mathrm{x}$ & & & \\
\hline 6 & Educación e Interculturalidad & & $x$ & & & \\
\hline 6 & Filosofía de la Educación: Contexto Latinoamericano & & $\mathrm{x}$ & & & \\
\hline 6 & Legislación Educativa & & $x$ & & & \\
\hline 6 & Realidad Educativa Ecuatoriana & & $\mathrm{x}$ & & & \\
\hline 7 & Educación y Género & & $x$ & & & \\
\hline 8 & Educación y Medio Ambiente & & $\mathrm{x}$ & & & \\
\hline 0 & Contexto e Introducción a la Carrera & & & & $x$ & \\
\hline 0 & Técnicas de Estudio & & & & $\mathrm{x}$ & \\
\hline 2 & Cosmovisión del Currículo & & & & $\mathrm{x}$ & \\
\hline 3 & Diseño y Evaluación de Proyectos & & & & $x$ & \\
\hline 4 & Ayudas Educativas & & & & $\mathrm{x}$ & \\
\hline 4 & Metodología de Elaboración Monográfica & & & & $x$ & \\
\hline
\end{tabular}


continuación Cuadro 3

\begin{tabular}{|c|c|c|c|c|c|c|}
\hline Nivel & Nombre de la asignatura & $\begin{array}{c}\text { Mat. } \\
\text { Pedag. }\end{array}$ & $\begin{array}{l}\text { Pedag. } \\
\text { Cien. }\end{array}$ & $\begin{array}{l}\text { Otras } \\
\text { Cienc. }\end{array}$ & $\begin{array}{l}\text { Metod. } \\
\text { Téc. }\end{array}$ & $\begin{array}{c}\text { Exp. } \\
\text { Aplicad. }\end{array}$ \\
\hline 5 & Métodos y Técnicas de Aprendizaje & & & & $\mathrm{x}$ & \\
\hline 5 & Planificación Curricular & & & & $\mathrm{x}$ & \\
\hline 7 & Evaluación Educativa & & & & $\mathrm{x}$ & \\
\hline 8 & $\begin{array}{l}\text { Diseño y Evaluación de Proyectos Educativos } \\
\text { Institucionales }\end{array}$ & & & & $\mathrm{x}$ & \\
\hline 8 & Metodología Elabo. Prod.Grado & & & & $\mathrm{x}$ & \\
\hline 9 & Denuncia Tesis & & & & $\mathrm{x}$ & \\
\hline 0 & Lenguaje y Pensamiento Lógico & & & $\mathrm{X}$ & & \\
\hline 1 & Comunicación Escrita & & & $\mathrm{X}$ & & \\
\hline 1 & Desarrollo del Pensamiento & & & $\mathrm{X}$ & & \\
\hline 1 & Psicología General & & & $\mathrm{X}$ & & \\
\hline 2 & Derechos de la Niñez & & & $\mathrm{X}$ & & \\
\hline 2 & Psicología del Desarrollo & & & $\mathrm{X}$ & & \\
\hline 3 & Antropología Cristiana & & & $\mathrm{X}$ & & \\
\hline 4 & Comunicación no Verbal & & & $\mathrm{X}$ & & \\
\hline 5 & Antropología Cultural & & & $\mathrm{X}$ & & \\
\hline 5 & Ética de la Persona & & & $\mathrm{X}$ & & \\
\hline 6 & Doctrina Social de la Iglesia & & & $\mathrm{X}$ & & \\
\hline 1 & Pedagogía General & $\mathrm{x}$ & & & & \\
\hline 2 & Corrientes Pedagógicas Contemporáneas & $\mathrm{x}$ & & & & \\
\hline 3 & Didáctica General & $\mathrm{x}$ & & & & \\
\hline 4 & Problemas del Aprendizaje & $\mathrm{x}$ & & & & \\
\hline 5 & Pedagogía Comparada & $\mathrm{x}$ & & & & \\
\hline 8 & Pedagogía Crítica & $\mathrm{x}$ & & & & \\
\hline 0 & Introducción a la UPS & & & & & $\mathrm{X}$ \\
\hline 2 & Electiva I & & & & & $\mathrm{X}$ \\
\hline 3 & Optativa I & & & & & $\mathrm{x}$ \\
\hline 4 & Electiva II & & & & & $\mathrm{x}$ \\
\hline 4 & Práctica I: Análisis de la Realidad Educativa & & & & & $\mathrm{X}$ \\
\hline 5 & Optativa II & & & & & $\mathrm{x}$ \\
\hline 6 & Práctica II:Inves.Docen.Consul.Ped. & & & & & $x$ \\
\hline 7 & Gerencia Educativa & & & & & $\mathrm{X}$ \\
\hline 7 & Pedagogía Liberadora & & & & & $\mathrm{X}$ \\
\hline 7 & Pedagogía Salesiana & & & & & $\mathrm{X}$ \\
\hline 8 & Investigación Educativa & & & & & $\mathrm{X}$ \\
\hline
\end{tabular}




\section{Cuadro 4}

Parvularia 2002

\begin{tabular}{|c|c|c|c|c|c|c|}
\hline Nivel & Nombre de la asignatura & $\begin{array}{l}\text { Mat. } \\
\text { Pedag. }\end{array}$ & $\begin{array}{l}\text { Pedag. } \\
\text { Cien. }\end{array}$ & $\begin{array}{l}\text { Otras } \\
\text { Cienc. }\end{array}$ & $\begin{array}{l}\text { Metod. } \\
\text { Téc. }\end{array}$ & $\begin{array}{l}\text { Exp. } \\
\text { Aplicad. }\end{array}$ \\
\hline 1 & Sistema Educativo Salesiano & & $x$ & & & \\
\hline 1 & Visión retrospectiva de la educación & & $x$ & & & \\
\hline 2 & Educación y Comunicación & & $x$ & & & \\
\hline 2 & Familia y Educación & & $x$ & & & \\
\hline 3 & Filosofía de la Educación & & $x$ & & & \\
\hline 3 & Psicología del Aprendizaje & & $x$ & & & \\
\hline 3 & Sociología de la Educación & & $x$ & & & \\
\hline 4 & Epistemología de la Educación & & $x$ & & & \\
\hline 4 & Infopedagogía & & $\mathrm{x}$ & & & \\
\hline 8 & Necesidades educativas especiales & & $x$ & & & \\
\hline 2 & Cosmovisión del Currículo & & & & $\mathrm{x}$ & \\
\hline 3 & Diseño y Evaluación de Proyectos & & & & $x$ & \\
\hline 4 & Ayudas Educativas & & & & $x$ & \\
\hline 4 & Metodología de Elaboración Monográfica & & & & $x$ & \\
\hline 5 & Metodología de la exp. Lúdica & & & & $x$ & \\
\hline 5 & Metodología Edu. de 0-4 años & & & & $x$ & \\
\hline 5 & Planificación Curricular & & & & $\mathrm{x}$ & \\
\hline 6 & Evaluación Educativa & & & & $x$ & \\
\hline 6 & Metodología del Lenguaje & & & & $x$ & \\
\hline 6 & Metodología Edu. de 4-7 años & & & & $\mathrm{x}$ & \\
\hline 6 & Metodología Matemática & & & & $x$ & \\
\hline 7 & Metodología Elaboración pro. licenciat & & & & $\mathrm{x}$ & \\
\hline 7 & Música y danza aplic. Pedal & & & & $\mathrm{x}$ & \\
\hline 7 & Teatro y Títeres y ap. pedag. & & & & $\mathrm{x}$ & \\
\hline 8 & Denuncia Tesis & & & & $x$ & \\
\hline 9 & Denuncia Tesis & & & & $x$ & \\
\hline 1 & Comunicación Escrita & & & $x$ & & \\
\hline 1 & Desarrollo del Pensamiento & & & $x$ & & \\
\hline 1 & Psicología General & & & $x$ & & \\
\hline 2 & Derechos de la Niñez & & & $x$ & & \\
\hline 2 & Psicología del Desarrollo & & & $x$ & & \\
\hline 3 & Antropología Cristiana & & & $x$ & & \\
\hline 4 & Comunicación no Verbal & & & $\mathrm{x}$ & & \\
\hline
\end{tabular}


continuación Cuadro 4

\begin{tabular}{|c|c|c|c|c|c|c|}
\hline Nivel & Nombre de la asignatura & $\begin{array}{l}\text { Mat. } \\
\text { Pedag. }\end{array}$ & $\begin{array}{l}\text { Pedag. } \\
\text { Cien. }\end{array}$ & $\begin{array}{l}\text { Otras } \\
\text { Cienc. }\end{array}$ & $\begin{array}{l}\text { Metod. } \\
\text { Téc. }\end{array}$ & $\begin{array}{l}\text { Exp. } \\
\text { Aplicad. }\end{array}$ \\
\hline 5 & Desarrollo Biológico & & & $\mathrm{x}$ & & \\
\hline 5 & Ética Profesional & & & $\mathrm{x}$ & & \\
\hline 5 & Expresión Grafoplástica & & & $\mathrm{x}$ & & \\
\hline 6 & Desarrollo Emocional & & & $\mathrm{x}$ & & \\
\hline 6 & Doctrina Social de la Iglesia & & & $x$ & & \\
\hline 7 & Educación Ambiental & & & $x$ & & \\
\hline 7 & Literatura Infantil & & & $\mathrm{x}$ & & \\
\hline 1 & Pedagogía General & $\mathrm{X}$ & & & & \\
\hline 2 & Corrientes Pedagógicas Contemporáneas & $x$ & & & & \\
\hline 3 & Didáctica General & $\mathrm{X}$ & & & & \\
\hline 4 & Problemas del Aprendizaje & $x$ & & & & \\
\hline 2 & Electiva I & & & & & $\mathrm{X}$ \\
\hline 3 & Optativa I & & & & & $\mathrm{X}$ \\
\hline 4 & Electiva II & & & & & $\mathrm{X}$ \\
\hline 4 & Práctica: Análisis de la Realidad Educativa & & & & & $\mathrm{X}$ \\
\hline 5 & Práctica con niños 0-4 años & & & & & $\mathrm{X}$ \\
\hline 6 & Práctica con niños 4-7 años & & & & & $\mathrm{X}$ \\
\hline 7 & Administración y Gestión Educativa & & & & & $\mathrm{X}$ \\
\hline 8 & Educación para el des. Psicomotriz & & & & & $\mathrm{X}$ \\
\hline 8 & Optativa II & & & & & $\mathrm{X}$ \\
\hline 8 & Práctica en Adm y G. & & & & & $\mathrm{x}$ \\
\hline
\end{tabular}

Un promedio de las seis especialidades de pedagogía:

- Educación general básica. CCSP

- Cultura y Estética

- Lenguaje y comunicación

- Matemáticas

- Parvularia

- Pedagogía

Muestra que las materias específicamente pedagógicas representan el 9\% del pensum o programa de la ca- rrera; las materias pedagógicas, pero tratadas desde otras ciencias o desde otros campos del conocimiento representa el 35\%; materias relativas a otras ciencias y campos de conocimiento, el 21\%; las materias metodológicas y técnicas, el 13\%; materias experimentales y aplicadas el 3.6\%.

Si se consideran los curriculum o programas de la carrera del año 2002 de EGB parvularia y EGB pedagogía, puede comprobarse una ligera modificación: las materias pedagógicas re- 
presentan el $28 \%$ del pensum, las materias referidas a la pedagogía desde otras ciencias o campos de conocimiento, el 23\%; las materias de metodología y técnicas, el $18 \%$; y las materias experimentales y aplicadas el $13 \%$.

Sin embargo, cuando se analizan los programas de las materias en sus contenidos detallados, proporcionando una información más precisa, el resultado se modifica sensiblemente en los programas del año 2002 de Pedagogía y Parvularia.

Según estos datos la carrera de pedagogía se basaría sobre una concepción científica de la pedagogía extremadamente reducida (entre un $11 \%$ y $7 \%$ ) de materias específicamente pedagógicas), y que trataría de compensar con una muy amplia área de conocimientos relativos a la educación en general o a los enfoques de la pedagogía desde las otras ciencias $(29 \%$ y $19 \%)$; o bien recurriendo a conocimientos de otras ciencias $(20 \%$ y $26 \%)$, a materias de carácter metodológico y técnico (21\% y $29 \%)$ y experimentales o aplicadas (19\% y $19 \%)$.

La cuestión que se plantea es si realmente existe una ciencia pedagógica suficientemente constituida o si la pedagogía constituye en realidad una ciencia fundada sobre una base teórica, con una epistemología propia, con un desarrollo relativamente amplio de conocimientos y orientaciones, campos de especialización y de aplicación de la ciencia, componentes todos estos propios de toda ciencia.

\section{Cuadro 5}

\begin{tabular}{|l|r||r|}
\hline EGB Pedagogía & \multicolumn{1}{c}{ No } & \multicolumn{1}{|c|}{} \\
\hline Materias pedagógicas & 6 & $11 \%$ \\
\hline Pedag. desde otras ciencias & 16 & $29 \%$ \\
\hline Otras ciencias & 11 & $20 \%$ \\
\hline Metodolog.y técnicas & 12 & $21 \%$ \\
\hline Experimentales y aplicadas & 11 & $19 \%$ \\
\hline TOTAL & 56 & 100 \\
\hline
\end{tabular}

\begin{tabular}{|l||r||r|}
\hline EGB Parvularia & \multicolumn{1}{c|}{ No $^{\circ} \%$} \\
\hline Materias pedagógicas & 4 & $7 \%$ \\
\hline Pedag. desde otras ciencias & 10 & $19 \%$ \\
\hline Otras ciencias & 14 & $26 \%$ \\
\hline Metodolog. y técnicas & 16 & $29 \%$ \\
\hline Experimentales y aplicadas & 10 & $19 \%$ \\
\hline TOTAL & 54 & 100 \\
\hline
\end{tabular}


Esta misma cuestión podría formularse desde la perspectiva académica y de la elaboración de un programa de estudios o currículo de pedagogía en cuanto ciencia, para transformarla en un programa de estudios y de formación. Tal precisión importa en la medida que lo realmente formativo, lo que forma científica y profesionalmente, no es tanto una acumulación de materias organizadas con una cierta coherencia, cuanto la organización y coherencia y desarrollo científicos de dichas materias. 